\title{
Divulgación
}

\section{Investigación en ciencias de la salud}

\section{Cantora, AM. • Corti, M.R. • Giuggia de Stratta, M.G.}

Escuela Superior de Sanidad. Facultad de Bioquímica y Ciencias

Biológicas. Universidad Nacional del Litoral. Ciudad Universitaria.

Paraje El Pozo s/n, 3000, Santa Fe, Argentina.

E-mail: afilippa2011@gmail.com

mcorti@fbcb.unl.edu.ar

romastratta@fibertel.com.ar

RESUMEN: Este trabajo se inscribe en el marco del Proyecto de Investigación y Desarrollo (CAI+D 2009) denominado "Ética profesional y Conocimiento en Ciencias de la Salud". Tiene como objetivo conocer los criterios que utilizan los profesionales de la salud para calificar a la investigación científica y vincularla con la toma de decisiones en la práctica. Es una investigación cualitativa, se diseñó una entrevista semiestructurada, organizada en cinco ejes. Se analizan dos de ellos que se refieren a "Áreas temáticas investigadas y su vinculación con problemas locales". Las preguntas estuvieron orientadas a conocer qué criterios prevalecen en las prácticas profesionales en referencia a problemas de salud, cómo se eligen las temáticas para la investigación en la Facultad de Bioquímica y Ciencias Bilógicas y en la Facultad de Ciencias Médicas y si se perciben las demandas en cuanto a problemas relacionados a la calidad de vida, considerando los determinantes sociales.

PALABRAS CLAVE: Ciencias de la salud, Investigación, Problemas locales, Determinantes sociales.

SUMMARY: Research in health sciences This work forms part of the Research and Development Project (CAI+D 2009) entitled "Professional Ethics and Knowledge in Health Sciences". The aims of this ongoing project are to determine the criteria used by health professionals to qualify scientific research and link it to decision-making in their practice. As a qualitative study, we designed a semistructured interview, organized into five areas. We analyzed two of them: "Thematic areas investigated and their connection to local problems". The questions were aimed to know what criteria take precedence over health problems, 
how to choose the topics for research in the School of Biochemistry and Biological Sciences and in the School of Medical Sciences, and if the demands are perceived as problems related the quality of life, considering social determinants.

KEYWORDS: Health sciences, Research, Local issues, Social determinants.

\section{Introducción}

"Suele pensarse que la medicina y la ética son esferas separadas del conocimiento, pertenecientes a campos bien diferenciados. La medicina, en su concepción moderna y occidental, es una rama de la ciencia natural, usa el método experimental y sus explicaciones se basan en la observación y el análisis objetivo de los hechos, mientras que la ética es una rama de las humanidades, su método de conocimiento es mucho más especulativo y no tiene como finalidad describir ni explicar los hechos, sino valorarlos. Sin embargo, la aparición de los determinantes sociales de la salud está contribuyendo a difuminar esa frontera, sobre todo en los campos de la epidemiología y la justicia social. Los determinantes sociales de la salud se refieren a los factores sociales (clase, género, edad, etnia...) que, respondiendo a un determinado contexto socioeconómico y político (que incluye, entre otros ámbitos, el mercado de trabajo, las políticas macroeconómicas y las políticas del estado de bienestar), afectan a las desigualdades de salud" (1).

La investigación sobre los determinantes sociales de la salud ha registrado un importante desarrollo demostrando la pertinencia de desplazar el enfoque de los problemas de salud desde los factores de riesgo individuales hacia los patrones sociales que configuran las posibilidades de la gente de ser saludable. No obstante y pese a la abruma- dora producción bibliográfica al respecto (2), la persistencia de los problemas de salud relacionados con condiciones estructurales injustas, tales como el hambre, la pobreza extrema y la exclusión social (algunos de los ítems que constituyen la "agenda inconclusa" de América Latina según la Organización Panamericana de la Salud (3) dan cuenta de la dimensión ético-política implicada en el desarrollo científico. Las mayores dificultades no están en el reconocimiento de estas relaciones de la salud con los modos de vida de las poblaciones sino en una cierta incapacidad en el plano de la salud colectiva para generar políticas sanitarias que den respuesta a esos problemas (4).

En todo el mundo, las personas socialmente desfavorecidas tienen menos acceso a los recursos sanitarios básicos y al sistema de salud en su conjunto. Es así como enferman y mueren con mayor frecuencia que aquellas que pertenecen a grupos que ocupan posiciones sociales más privilegiadas. Esto se hace más crítico en algunos de los grupos más vulnerables. Estas inequidades han aumentado a pesar de que nunca antes han existido en el mundo la riqueza, los conocimientos y la sensibilidad e interés por los temas que atañen a la salud como en la actualidad.

"Paradójicamente, existe suficiente evidencia, particularmente proveniente de países desarrollados, de acciones posibles 